\title{
Surgical prevention of Lymphedema following lymph node dissection: LY.M.P.H.A. technique.
}

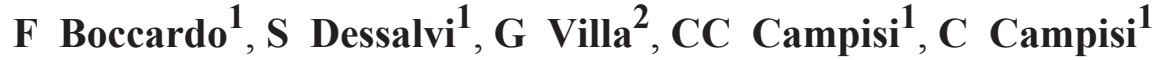 \\ ${ }^{1}$ IRCCS Ospedale Policlinico San Martino. Department of Surgery - Unit of Lymphatic Surgery. University of Genoa, \\ Largo R. Benzi 10, 16132, Genoa, Italy. \\ 2 IRCCS Ospedale Policlinico San Martino. Unit of Nuclear Medicine, Largo R. Benzi 10, 16132, Genoa, Italy.
}

submitted: Feb 23, 2018, accepted: Mar 19, 2018, EPub Ahead of Print: Mar 28, 2018, published: Jun 30, 2018

Conflict of interest: None

DOI: 10.24019/jtavr.41 - Corresponding author: Prof. Francesco Boccardo, francesco.boccardo@unige.it

(C) 2017 Fondazione Vasculab impresa sociale ONLUS. All rights reserved.

\begin{abstract}
Background

A side-effect of axillary lymph node excision and radiotherapy for breast cancer is arm lymphedema in about $25 \%$ patients (ranging from 13 to $52 \%$ ). Sentinel lymph node (SLN) biopsy has reduced the severity of swelling to nearly $6 \%$ patients (from 2 to $7 \%$ ) and, in case of positive SLN, complete axillary dissection (AD) is still required. That is why Axillary Reverse Mapping method (ARM) was developed aiming at identifying and preserve lymphatics draining the arm. Leaving in place lymph nodes related to arm lymphatic drainage would decrease the risk of arm lymphedema, but not retrieving all nodes, the main risk is to leave metastatic disease in the axilla. Based on long term experience in lymphatic-venous anastomoses (LVA) for lymphedema treatment, Authors conceived and carried out preventive LVA during nodal dissection (Lymphatic Microsurgical Preventing Healing Approach - LY.M.P.H.A. technique).

Methods

78 patients underwent axillary nodal dissection for breast cancer treatment and in 74 of them LY.M.P.H.A. procedure was performed. Indications to LY.M.P.H.A. technique were based on clinical and lymphoscintigraphic parameters. All blue nodes were resected and 2 to 4 main afferent lymphatics from the arm could be prepared and used for anastomoses. Lymphatics were introduced inside the vein cut-end by a U-shaped stitch. Volumetry was performed
\end{abstract}

preoperatively in all patients and after 1, 6, 12 months and once a year. Lymphoscintigraphy was performed in 45 patients preoperatively and in 30 also postoperatively after at least over 1 year.

Results

Seventy-one patients had no sign of lymphedema. In 3 patients, lymphedema occurred after 8-12 months postoperatively. The incidence of secondary arm lymphedema after LY.M.P.H.A. technique was therefore $4.05 \%$.

\section{Conclusion}

LVA proved not only to prevent lymphedema but also to reduce early lymphatic complications (i.e. lymphorrhea, lymphocele). LY.M.P.H.A. technique is also useful in patients with melanoma of the trunk and vulvar cancer, in whom it is possible to perform preventive LVA simultaneously with inguinal lymphadenectomy. Lymphedema is a consequence of cancer treatment. The use of the blue dye and of LVA helps to solve the problem of preventing secondary arm and leg lymphedema. LY.M.P.H.A. represents a rational approach to the prevention of lymphedema following axillary and groin surgery in the therapy of breast cancer, melanoma, vulvar cancer and other tumors.

Keywords Lymphedema, lymph node dissection, prevention, LY.M.P.H.A. technique, lymphatic-venous anastomosis. 

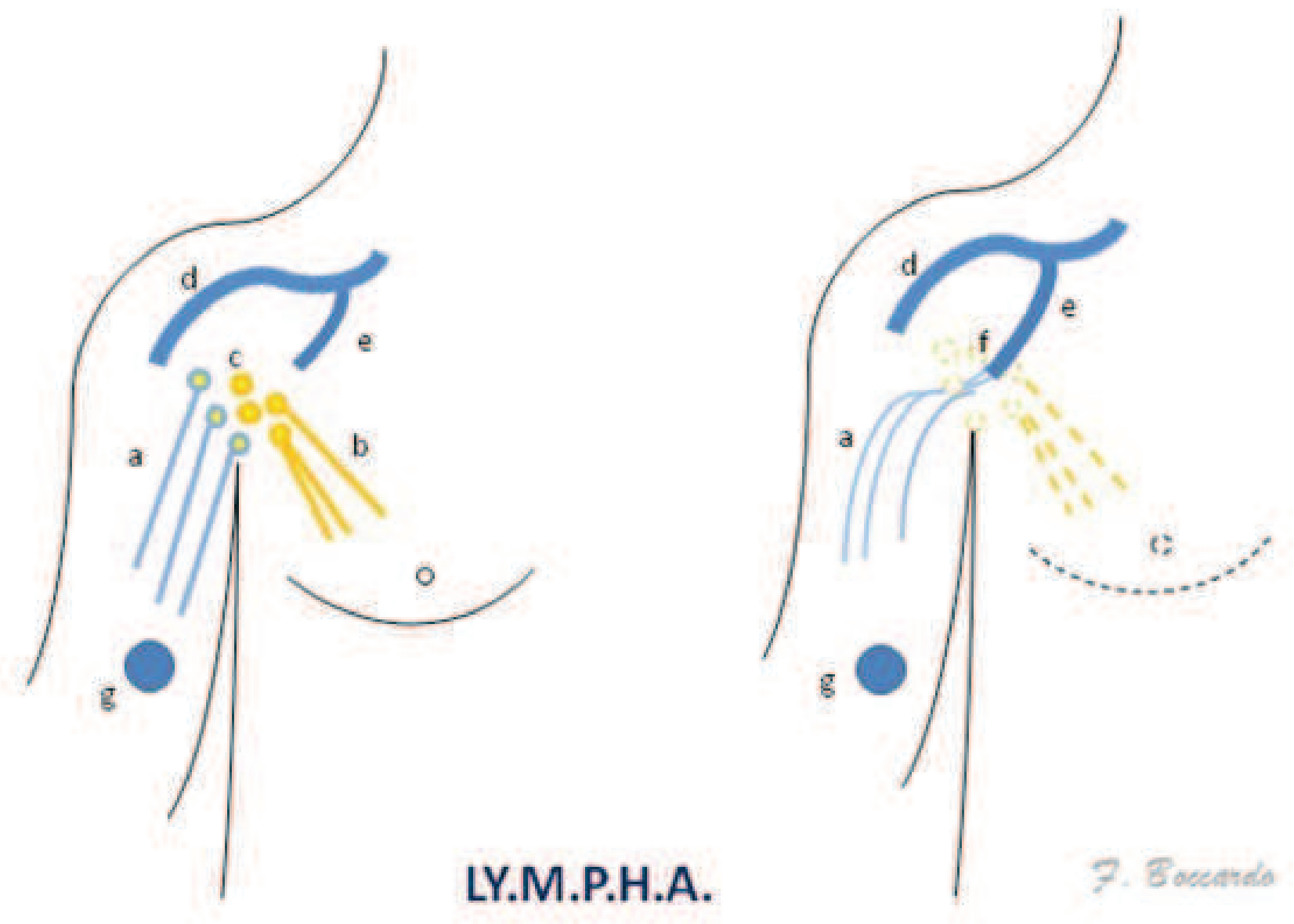

Figure 1 - Schematic drawing of LY.M.P.H.A. procedure at the axilla. a) Arm lymphatics. b) Breast lymphatics. c) Axillary nodes. d) Axillary vein. e) Tributary vein. f) Lymphatic venous anastomosys. g) Blue dye.

\section{Introduction}

A side-effect of axillary lymph node excision and radiotherapy for breast cancer is arm lymphedema in about $25 \%$ patients (ranging from 13 to $52 \%$ ). Sentinel lymph node (SLN) biopsy has reduced the severity of swelling to nearly $6 \%$ patients (from 2 to $7 \%$ ) and, in case of positive SLN, complete axillary dissection (AD) is still required. That is why Axillary Reverse Mapping method (ARM) ${ }^{1,2}$ was developed aiming at identifying and preserve lymphatics draining the arm. It consists in injecting intradermally and subcutaneously a small quantity (1-2 $\mathrm{ml}$ ) of blue dye at the medial surface of the arm which helps in locating the draining arm lymphatic pathways. ARM technique allowed to find variable clinical anatomical conditions from what was already generally known, that is the most common location of arm lymphatics below and around the axillary vein. In about one-third of the cases, blue lymphatics can be found till $3-4 \mathrm{~cm}$ below the vein, site where SLN can easily be located, justifying the occurrence of lymphedema after only SLN biopsy ${ }^{3}$. ARM procedure showed that blue nodes were almost always placed at the lateral part of the axilla, under the vein and above the second intercostal brachial nerve ${ }^{4}$. Leaving in place lymph nodes related to arm lymphatic drainage would decrease the risk of arm lymphedema, but not retrieving all nodes, the main risk is to leave metastatic disease in the axilla. Conversely, arm lymphatic pathways when they enter the axilla, cannot be site of breast tumoral disease and their preservation would certainly bring about a significant decrease of lymphedema occurrence rate. Based on long term experience in lymphaticvenous anastomoses (LVA) ${ }^{5,6}$ for lymphedema treatment, Authors conceived and carried out preventive LVA during nodal dissection. Patients candidate to the preventive procedure were selected by body mass index (BMI) and lymphoscintigraphy transport Index (TI): indications were represented by $>30 \mathrm{BMI}$ and $>10$ TI. Authors report long term outcome of their experience in the prevention of breast cancer related lymphedema using 
Lymphatic Microsurgical Preventing Healing Approach (LY.M.P.H.A.), which consists in anastomosing arm lymphatics to a tributary branch of axillary vein ${ }^{7}$.

\section{Patients and Methods}

From July 2008 to December 2012, 78 patients underwent axillary nodal dissection for breast cancer treatment and in 74 of them LY.M.P.H.A. procedure was performed (Table I). In 4 patients LY.M.P.H.A. could not be carried out because no afferent lymphatics could be found ( 3 patients) and because of massive metastatic disease (1 patient). Indications to LY.M.P.H.A. technique were based on clinical and lymphoscintigraphic parameters. It is well known that obesity predispose to lymphedema and therefore patients with BMI higher than 30 are considered at high risk for lymphedema and are candidates for LY.M.P.H.A.. Patients with normal BMI could be studied with lymphoscintigraphy which was able to point out latent lymphatic impairment (TI > 10), still not evident clinically.

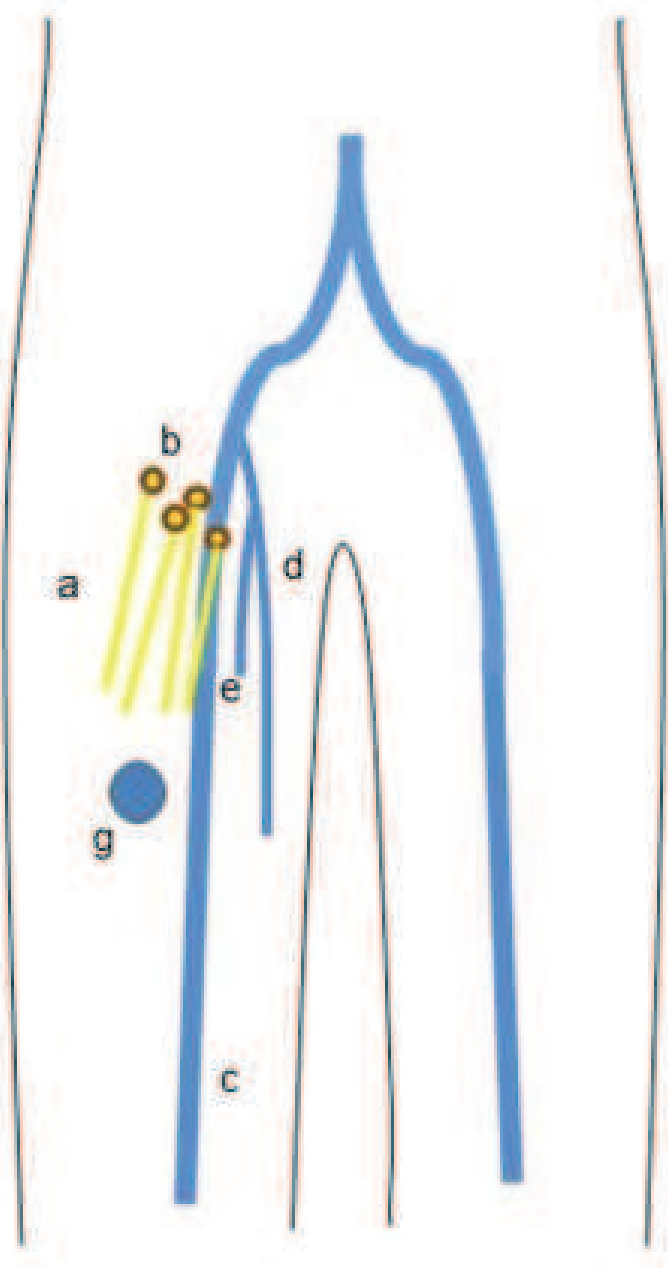

LY.M.P.H.A.

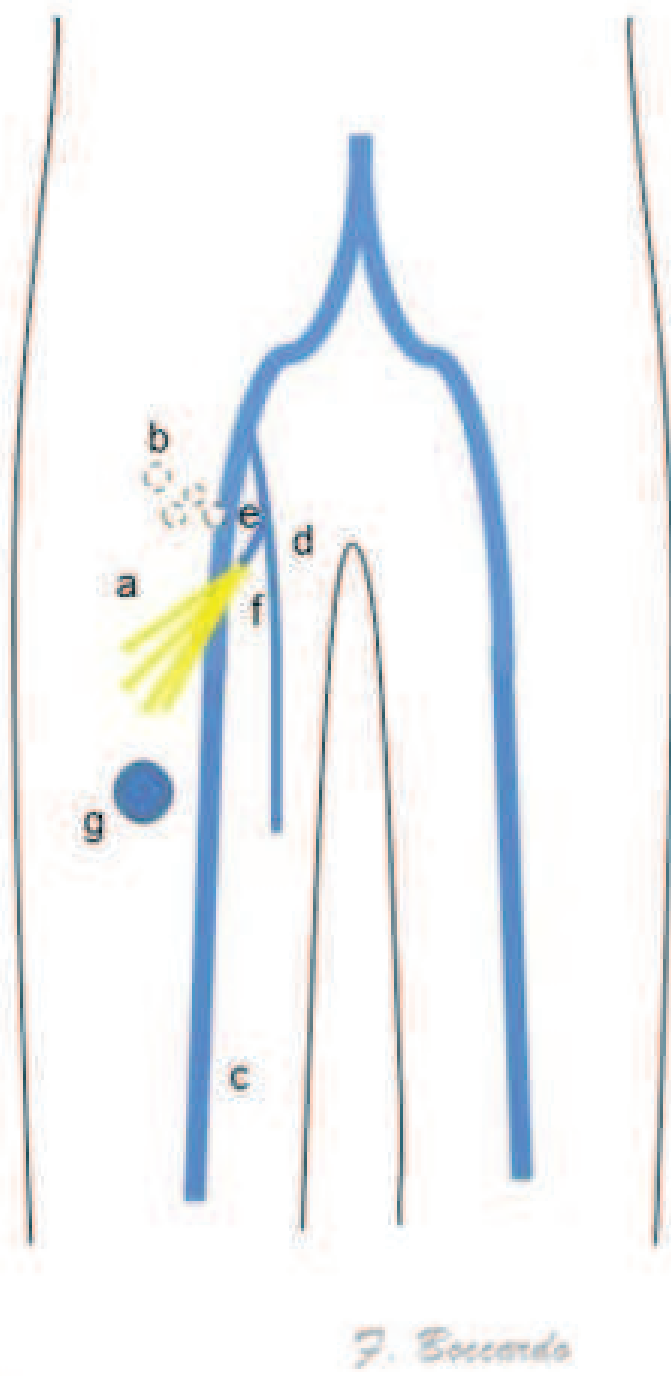

Figure 2 - Schematic drawing of LY.M.P.H.A. procedure at the groin. a) Leg lymphatics. b) Groin nodes. c) Femoral vein. d) Great saphenous vein. e) Tributary vein. f) Lymphatic venous anastomosys. g) Blue dye.

Blue nodes in relation to lymphatic arm drainage could be therefore identified in almost all patients after blue dye injection at the arm. All blue nodes were resected and 2 to 4 main afferent lymphatics from the arm could 
be prepared and used for anastomoses. Lymphatics were introduced inside the vein cut-end by a U-shaped stitch. Other few stitches were given to fix the lymphatic adventitia to the vein wall (Figs.1-4). The operation usually takes only 20 minutes averagely, since both lymphatics and the vein are prepared during nodal dissection. Drain tubes were removed after about 10 days (7-12 days). No bandages at the arm are necessary and patients do not wear any elastic garment.

Volumetry was performed preoperatively in all patients and after 1, 3, 6, 12 months and once per year. Lymphoscintigraphy was performed in 45 patients preoperatively and in 30 also postoperatively after at least over 1 year, comparing pre-op and post-op lymph $\mathrm{TI}^{8}$. Statistical analysis was performed by Fisher's exact test ( $\mathrm{P}$ value $<0.05)$.

\section{Results}

Seventy-one patients had no sign of lymphedema and volumetry was coincident to preoperative condition (Table II). In 3 patients belonging to the initial clinical experience lymphedema occurred after 8-12 months postoperatively, usually with the appearance of lymphangitic attacks. Radiotherapy caused temporary edema of the arm in 8 patients and in 3 of them edema became permanent. The incidence of secondary arm lymphedema after LY.M.P.H.A. technique was therefore almost $4 \%$.

Lymphoscintigraphy showed the patency of lymphatic-venous anastomoses at 1-4 years after operation. Comparing lymphatic Transport Index to preoperative conditions, results showed a significant improvement in all patients with early liver tracer uptake. Lymphoscintigraphy was always done in the same department, studying superficial lymphatic circulation, by injecting the tracer at the dorsum of the hand.

\section{Discussion}

LVA proved not only to prevent lymphedema but also to reduce early lymphatic complications (i.e. lymphorrhea, lymphocele - no cases compared with the percentage reported in the literature: $4-89 \%$ ) thanks to the normal regional lymphatic flow.

An important aspect to point out is that, in the axilla, new lymphatic vessel formation (lymphangiogenesis) occurs in response to the ligation of lymphatic vessels involved in lymph node retrievement. Lymphangiogenesis and lymphatic hypertension were demonstrated experimentally in case of lymphatic drainage obstruction. And, in response to lymphatic hypertension, lympho-venous shunts open and provide alternative lymphatic pathways when the main ones are obstructed.

These mechanisms represent an adaptive response to lymphatic hypertension but are not enough to restore normal flow parameters. Furthermore, chronic obstruction to lymph flow progressively leads to a reduced lymphatic contractility, lymphatic thrombosis and fibrotic changes, at a different degree according to variable constitutional predisposition 12,13 .

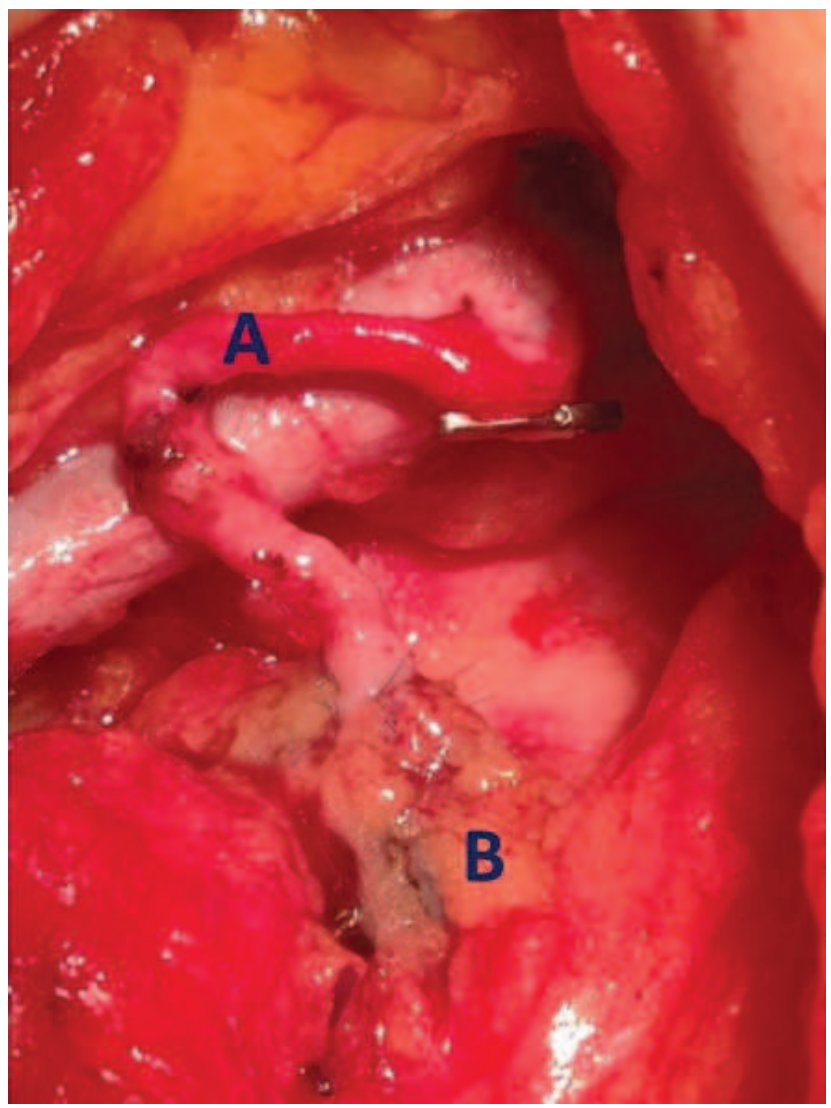

Figure 3 - LY.M.P.H.A. technique at the axilla. A Tributary of the axillary vein. B - Brachial lymphatics colored in blue.

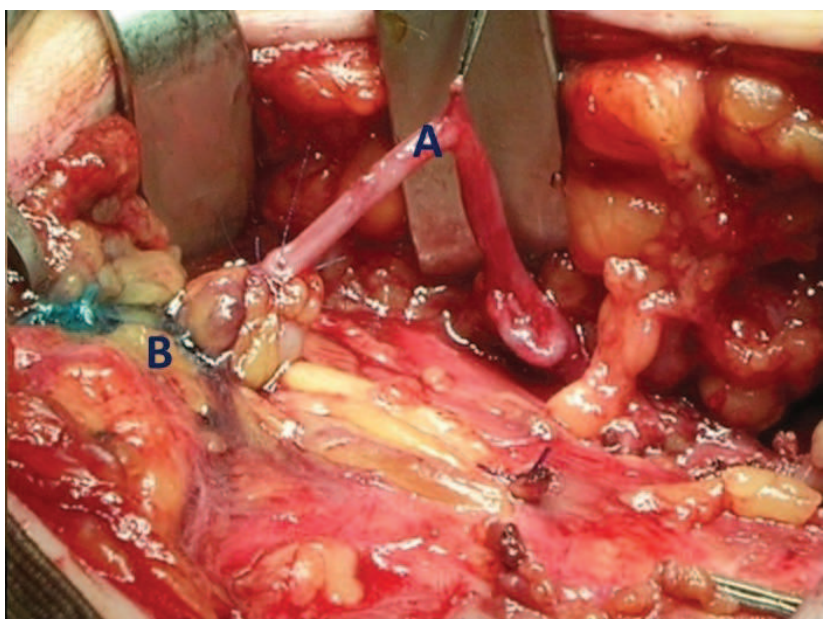

Figure 4 - LY.M.P.H.A. technique at the groin. A - Tributary of the great saphenous vein. B - Lower limb lymphatics colored in blue. 


\begin{tabular}{|lc|}
\hline Variable & Mean (range) \\
Age (Years) & $57(42-69)$ \\
Body Mass Index & $24(21-33)$ \\
Variable & \#cases (range) \\
& \\
Lymph Nodes Retrieved & $19(12-21)$ \\
Metastatics Lymph Nodes & $3(0-4)$ \\
Type of surgery (mastectomy/tumorectomy, cases) & $34 / 40$ \\
Radiotherapy (yes/no, cases) & $35 / 39$ \\
Cellulitis (yes/no, cases) & $14 / 60$ \\
Table I- Patients characteristics & \\
\hline
\end{tabular}

\begin{tabular}{|c|c|c|c|c|c|c|}
\hline \multirow[t]{3}{*}{ \# cases } & \multicolumn{3}{|c|}{ Volumetry - Mean $(\sigma)$} & \multicolumn{3}{|c|}{ Lymphoscintigraphy TI - Mean $(\sigma)$} \\
\hline & \multirow[t]{2}{*}{ Pre-Op } & \multicolumn{2}{|c|}{ Post-Op } & \multirow[t]{2}{*}{ Pre-Op } & \multicolumn{2}{|c|}{ Post-Op } \\
\hline & & 1 year & 4 years & & 1 year & 4 years \\
\hline 71 & $760(90)$ & $760(100)$ & $760(90)$ & $12(4)$ & $9(4)$ & $8(4)$ \\
\hline 3 & $770(120)$ & $104(2)$ & i & $14(4)$ & $16(4)$ & i \\
\hline \multicolumn{7}{|l|}{74 total } \\
\hline \multicolumn{7}{|c|}{ Table II - Clinical and instrumental assessment of results after LY.M.P.H.A procedure. } \\
\hline \multicolumn{7}{|c|}{$\begin{array}{l}\sigma \text { standard deviation. } P \text { value }<0.05 \text { (Pre-Op and Post-Op volumetric measurements in } 71 \text { patients). TI: Transport } \\
\text { Index. }\end{array}$} \\
\hline
\end{tabular}

Recent advances in the treatment of breast cancer, specifically as concerns the prevention of lymphatic complications following sentinel lymph node biopsy and axillary dissection brought to the proposal of a new technique to primarily prevent lymphedema by microsurgical lymphatic-venous anastomoses (LY.M.P.H.A.). L-V shunts have been chosen for LY.M.P.H.A. technique instead of lymph node or lymph vessels transplantation because it is a faster technique and does not require lymph node or lymph vessels harvesting ${ }^{14}$, 15. ARM technique allows to identify arm lymphatics and lymph nodes which can therefore be preserved even though there is the risk to leave undetected metastatic disease in the axilla. ARM is effective for sentinel lymph node biopsy because it allows to preserve brachial nodes when they are not involved in the procedure. But, it is almost impossible to preserve efferent lymphatics from the blue nodes because they join the common axillary nodal basin draining the breast. Thus, not preserving efferent lymphatics makes practically impossible to preserve arm lymphatic flow. So, on the basis of our wide experience in the treatment of lymphedema by microsurgical lymphaticvenous anastomoses (LVA), we thought to perform LVA immediately after finishing nodal axillary excision. The surgical technique proposed for patients with operable breast cancer requiring an axillary dissection consisted in carrying out LVA between arm lymphatics identified by injecting blue dye in the arm and an axillary vein branch simultaneously. It is almost always possible to find blue lymphatics and also to find a vein branch long enough to be connected to arm lymphatics which are usually locate very laterally.

Disruption of the axillary nodes and closure of arm lymphatics can explain the significantly high risk of early and late lymphatic complications after axillary dissection, especially the most serious complication, that is arm lymphedema ${ }^{16}$. Patients candidate to the preventive procedure were selected by BMI and lymphoscintigraphy TI: indications were represented by $>30 \mathrm{BMI}$ and $>$ $10 \mathrm{TI}$. TI is a semi quantitative parameter that derives from the measurements of the tracer clearance from the injection point, lymph node uptake and pattern of the drainage along the limb. The incidence of secondary arm lymphedema after LY.M.P.H.A. technique was therefore $4.05 \%$, compared to the incidence rate after only complete 
axillary dissection reported in the literature which ranges from 13 to $65 \%$, depending on the criteria used and means of assessment. Finally, it is important to underline that all three patients who developed lymphedema notwithstanding LY.M.P.H.A. technique belonged to the preliminary clinical experience.

Based on our experience and of the experience of other groups, we can say that the learning curve for LY.M.P.H.A. is of about 20-30 cases. There is no particular method on which we have based to say that but this suggestion derives from the direct clinical experience. LY.M.P.H.A. technique is also useful in patients with melanoma of the trunk, in whom it is possible to perform preventive lymphatic- venous anastomosis (LVA) simultaneously with inguinal lymphadenectomy. The blue dye is injected at the medium third of the medial surface of the thigh. Blue lymphatics afferent to the groin are isolated and anastomosed to a tributary of the great saphenous vein. In most cases, the medial accessory saphenous vein is used and all blue afferent lymphatics are anastomosed to the same vein segment (multiple LVA). A shirtsleeve-shape technique is used. This procedure permits the lymph coming from the main lymphatic pathways of the limb to flow into the bloodstream.

This preventive surgical approach can only be carried out in patients with melanoma localized at the trunk.

In case of melanoma of the extremities, this preventive approach cannot be used for oncological reasons and lymphedema development can be limited with accurate post-operative management. Immediate postoperative elastic wraps have proved their efficacy when placed from the foot to above the knee with multiple layers, replaced afterwards by compression stockings. Leg elevation is useful as well when the patient is still lying on the bed.

After an accurate oncological assessment and the absence of any sign of relapse of the pathology, lymphedema can be treated afterwards by lymphatic

\section{References}

1) Thompson M, Korourian S, Henry-Tillman R, Adkins L, Mumford S, Westbrook KC, Klimberg VS. Axillary Reverse Mapping (ARM): A New Concept to Identify and Enhance Lymphatic Preservation. Ann Surg Oncol, 2007; 14(6):1890-1895.

2) Sakorafas GH, Perosa G, Cataliotti L, Vlastos G. Lymphedema Following Axillary Lymph Node Dissection For Breast Cancer. Surg Oncol, 2006; 15(3):153-165.

3) Nos C, Lesieur B, Clough KB, Lecuru F: Blue Dye Injection in the Arm in Order to Conserve the Lymphatic Drainage of the Arm in Breast Cancer Patients Requiring an Axillary Dissection. Annals of Surgical Oncology 14(9):2490-2496 microsurgery, in order to bypass the obstacle to lymph flow by derivative multiple LVA at the groin. In these patients, lymphoscintigraphy represents the gold standard to assess lymphatic transport capacity preoperatively and afterwards to evaluate long term results.

Venous ultrasonography is performed in all patients to study vein circulation. LVA proved to be successful above all in lymphedema at early stages, due to the absence of fibrotic changes of tissues and lymphatic wall.

A correctly performed completion lymphadenectomy with careful attention to surgical details can help to minimize wound complications, decrease the time of closed-suction drains and decrease venous thromboembolism. As concern lymphedema conversely, there is always a congenital predisposition even though it appears to be a real secondary lymphedema in terms of anatomical-functional predisposition. Risk patients may be assessed preoperatively with lymphoscintigraphy and be addressed to preventive measures ${ }^{17,18}$.

Similar experiences have been reported in the literature, confirming the efficacy of LY.M.P.H.A. procedure in the prevention of secondary arm lymphedema and LY.M.P.H.A. technique was included among the surgical procedures to prevent breast cancer related lymphedema ${ }^{19-22}$.

\section{Conclusion}

Lymphedema is a consequence of cancer treatment and patients should be informed of the signs, symptoms and possibilities of prevention for lymphedema. The use of the blue dye and of LVA helps to solve the problem of preventing secondary arm and leg lymphedema even maintaining the oncological radicality. LY.M.P.H.A., therefore, might represent a rational approach to the prevention of lymphedema and reduce other lymphatic complications after axillary and groin surgery in the therapy of breast cancer, melanoma and other tumors.

4) Ponzone R, Mininanni P, Cassina E, Sismondi P. Axillary Reverse Mapping in Breast Cancer: Can we Spare what we Find? Ann Surg Oncol, 2007.

5) Campisi C, Bellini C, Campisi CC, Accogli S, Bonioli E, Boccardo F. Microsurgery for lymphedema: clinical research and longterm results. Microsurgery. 2010 May;30(4):256-60.

6) Auba C, Marre D, Rodriguez-Losada G, Hontanilla B. Lymphaticovenular anastomoses for lymphedema treatment: 18 months postoperative outcomes. Microsurgery 2012 May; 32 (4):261-8.

7) Boccardo F, Casabona F, De Cian F, Friedman D, Villa G, Bogliolo S, Ferrero S, Murelli F, Campisi C. Lymphedema Microsurgical Preventive Healing Approach: A New Technique For 
Primary Prevention Of Arm Lymphedema After Mastectomy. Ann Surg Oncol 2009;16:703-708.

8) E. Kleinhans, R.G.Baumeister, D.Hahn, S.Siuda, U.Bull, E.Moser. Evaluation of transport kinetics in lymphoscintigraphy: follow up study in patients with translplanted lymphatic vessels. Eur J Nucl Med, 1985

9) Boccardo F, Fulcheri E, Villa G, Molinari L, Campisi C, Dessalvi S, Murdaca G, Campisi C, Santi PL, Parodi A, Puppo F, Campisi C. Lymphatic microsurgery to treat lympedema: techniques and indication for better results. Ann Plast Surg, 2013; Mar 28 (Epub).

10) Deutsch M, Land S, Begovic M, Sharif S. The Incidence Of Arm Edema In Women With Breast Cancer Randomized On The National Surgical Adjuvant Breast And Bowel Projet Study B-04 To Radical Mastectomy Versus Total Mastectomy And Radiotherapy Versus Total Mastectomy Alone. Int J Radiat Oncol Biol Phys, 2008; 70:1020-1024.

11) Kwan ML, Darbinian J, Schmitz KH, Citron R, Partee P, Kutner SE, Kushi LH. Risk Factors For Lymphedema In A Prospective Breast Cancer Survivorship Study: The Pathways Study. Arch Surg, 2010; 145:1055-1063.

12) Gartner R, Jensen MB, Kronborg L, Ewertz M, Kehlet H, Kroman N. Self-Reported Arm-Lymphedema And Functional Impairment After Breast Cancer Treatment: A Nationwide Study Of Prevalence And Associated Factors. Breast, 2010; 19:506-515.

13) Jila A, Kim H, Nguyen VP, Dumont DJ, Semple J, Armstrong D, Seto E, Johnston M. Lymphangiogenesis Following Obstruction Of Large Postnodal Lymphatics In Sheep. Microvasc Res 2007; 73:214-223.

14) Modi S, Stanton AWB, Svensson WE, Peters AM, Mortimer PS, Levick JR. Human Lymphatic Pumping Measured In Healthy And Lymphoedematous Arms By Lymphatic Congestion Lymphoscintigraphy. J Physiol 2007; 583.1:271-285.

15) Felmerer G, Sattler T, Lohrmann C, Tobbia D. Treatment of various secondary lymphedemas by microsurgical lymph vessel transplantation. Microsurgery 2012 Mar; 32 (3):171-7.
16) Francissen CMTP, Dings PJM, Van Dalen T, Strobbe LJA, Van Laarhoven HWM, De Wilt JHW: Axillary Recurrence After A Tumor-Positive Sentinel Lymph Node Biopsy Without Axillary Treatment: A Review Of The Literature. Ann Surg Oncol, 2012; 19:4140-4149.

17) Paskett ED, Dean JA, Oliveri JM, Harrop P. Cancer-Related Lymphedema Risk Factors, Diagnosis, Treatment, And Impact: A Review. J Clin Onc 2012; 30 (30):3726-3733.

18) Witte MH, Dellinger MT, Papendieck CM, Boccardo F. Overlapping biomarkers, pathways, processes and syndromes in lymphatic development, growth and neoplasia. Clin Exp Metastasis. 2012 Oct; 29(7):707-27.

19) Feldman S, Bansil H, Ascherman J, Grant R, Borden B, Henderson P, Ojo A, Taback B, Chen M, Ananthakrishnan P, Vaz A, Balci F, Divgi CR, Leung D, Rohde C. Single Institution Experience with Lymphatic Microsurgical Preventive Healing Approach (LYMPHA) for the Primary Prevention of Lymphedema. Ann Surg Oncol. 2015 Oct;22(10):3296-301.

20) Hahamoff M, Gupta N, Munoz D, Lee BT, Clevenger P, Shaw C, Spiguel L, Singhal D. A lymphedema surveillance program for breast cancer patients reveals the promise of surgical prevention. J Surg Res. 2018 Feb 1. pii:S0022-4804(17)30662-5.

21) McLaughlin SA, Staley AC, Vicini F, Thiruchelvam P, Hutchison NA, Mendez J, MacNeill F, Rockson SG, DeSnyder SM, Klimberg S, Alatriste M, Boccardo F, Smith ML, Feldman SM. Considerations for Clinicians in the Diagnosis, Prevention, and Treatment of Breast Cancer-Related Lymphedema: Recommendations from a Multidisciplinary Expert ASBrS Panel : Part 1: Definitions, Assessments, Education, and Future Directions. Ann Surg Oncol. 2017 Oct;24(10):2818-2826.

22) McLaughlin SA, DeSnyder SM, Klimberg S, Alatriste M, Boccardo F, Smith ML, Staley AC, Thiruchelvam PTR, Hutchison NA, Mendez J, MacNeill F, Vicini F, Rockson SG, Feldman SM. Considerations for Clinicians in the Diagnosis, Prevention, and Treatment of Breast Cancer-Related Lymphedema, Recommendations from an Expert Panel: Part 2: Preventive and Therapeutic Options. Ann Surg Oncol. 2017 Oct;24(10):2827-2835. 